\title{
Effects of gestational diabetes on junctional adhesion molecules in human term placental vasculature
}

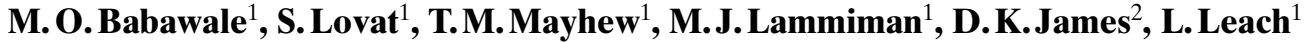 \\ ${ }^{1}$ School of Biomedical Sciences, Faculty of Medicine and Health Sciences, University of Nottingham, UK \\ ${ }^{2}$ School of Human Development, Faculty of Medicine and Health Sciences, University of Nottingham, UK
}

\section{Abstract}

Aims/hypothesis. The aim of this study was to investigate whether gestational diabetes mellitus, which occurs in the microvascular remodelling phase of placental development, causes alterations in surface expression of tight and adherens junctional molecules involved in endothelial barrier function and angiogenesis.

Methods. Term placenta, delivered by elective Caesarian section, from normal pregnancy $(n=5)$ and those complicated by gestational diabetes $(n=5)$ were perfusion-fixed and analysed by indirect immunofluorescence and confocal scanning microscopy. Using systematic random sampling, the surface expression of endothelial junctional proteins and the relative incidences of immunostained vessels were compared between the two study groups. Total vessel lengths were measured by stereological techniques. Results. The adherens junctional molecules, vascularendothelial cadherin and $\beta$-catenin, and the tight junctional molecules, occludin and zonula occludens-1 were localised to paracellular clefts in both study groups. The diabetic placentae showed pronounced reductions in the intensity of immunofluorescence and in the number of immuno-positive vessels. A corresponding statistically significant increase (from $19 \%$ to $56 \%$ ) in the percentage of vessels showing junctional anti-phosphotyrosine immunoreactivity was found. The differences observed represented real changes in the absolute lengths of immunostained regions along the vessels. The stereological measurements failed to detect any statistically significant change in the combined length of fetal vessels in gestational diabetic placenta.

Conclusion/interpretation. Our results suggest that even short duration diabetic insult, alters the surface expression of placental junctional proteins. This alteration could be mediated by the tyrosine-phosphorylation pathway. The changes suggest impaired barrier function rather than accelerated vascular growth. [Diabetologia (2000) 43: 1185-1196]

Keywords Endothelium, VE-cadherin, $\beta$-catenin, occludin, ZO-1, gestational diabetes, placenta
The human placenta is a highly vascularised organ whose major functions are to allow materno-fetal exchange of solutes and oxygen. Formation of the pla-

Received: 9 May 2000 and in revised form: 20 June 2000

Corresponding author: Dr L. Leach, E Floor, School of Biomedical Sciences, Queens Medical Centre, Nottingham NG7 2UH, UK.

Abbreviations: GDM, Gestational diabetes mellitus; VE-cadherin, vascular endothelial cadherin; ZO-1, zonula occludens 1; VEGF, vascular endothelial growth factor. cental fetal vessels (vasculogenesis) occurs in the first 4 weeks of pregnancy and elaboration of the microvascular beds (angiogenesis) occurs in the last trimester of pregnancy. The structure and permeability of these vessels, at term, resemble those of skeletal muscle capillaries [1,2]. Both vascular remodelling and effective barrier formation are key endothelial functions, which influence fetal well-being and perinatal outcome. One of the major complications in pregnancies is diabetes mellitus. In pregnancies in women with pre-existing diabetes, both vasculogenesis and angiogenesis, in theory, can be affected. Ges- 
tational diabetes (GDM) is however, by definition a complication arising during pregnancy [3], typically in the second or third trimester, after vasculogenesis has occurred. Thus, the GDM placental vascular bed could be an extremely valuable model for the study of microangiopathy in early diabetes because disturbances in vasculogenesis and pre-existing maternal microvascular disease affecting the uterine circulation are excluded.

The maternal glucose intolerance of GDM is usually mild but nevertheless is associated with a high incidence of complications during pregnancy including excessive fetal growth (macrosomia), increased perinatal mortality and morbidity of the infants [3]. An early study of the placenta from gestational diabetic mothers found there was statistically significantly more surface area of exchange between mother and fetus, in terms of peripheral villous and capillary surface areas [4]. These changes were hypothesised to be structural modifications that represent successful adaptations to maternal metabolic disturbances. The cellular mechanisms involved in placental vascular remodelling in GDM are however, not known.

Diabetes mellitus has been linked to accelerated microangiopathy; both capillary hypertension and inherited or acquired changes in capillary permeability have been associated with this [5]. Increased angiogenesis is also a common feature of diabetic vasculopathy, specifically manifest in diabetic retinopathy [6]. In placenta from pregnancies complicated by pre-existing diabetes, various pathological alterations, including placental hypertrophy and hypovascularity have been reported $[7,8]$. Increases in the volume, surface area and length of placental capillaries in the well-controlled diabetic mother have also been reported and these changes are accompanied by greater total diffusive conductance [9]. The integrity of the placental endothelium and the mechanisms which allow increased angiogenesis in these pregnancies are still not clear.

The endothelial paracellular cleft is one of the principal pathways for the passage of hydrophilic solutes, acting as the structural equivalent of the "small pore" [10] specifically in continuous endothelia, such as those in guinea-pig [11] and human placenta [2]. Two physical entities in the pathway, the tight and adherens junctions are thought to regulate both cell-cell adhesion and permeability. It is the adhesive molecules present within these junctional complexes which dictate the permeability status of the endothelium.

Vascular endothelial cadherin (VE-cadherin) is the transmembrane glycoprotein of endothelial adherens junctions. Homotypic binding of its extracellular portion allows cell-cell adhesion whereas rigidity of the extracellular domains and clustering behaviour might influence adhesive strength [12]. The cytoplasmic tail of VE-cadherin is linked to signal transduc- tion ligands of the armadillo family, $\beta$-catenin, plakoglobin $(\gamma$-catenin) and $\mathrm{p} 120$; binding with $\alpha$-catenin allows anchorage to perijunctional actin microfilaments [13]. Tyrosine phosphorylation of VE-cadherin and $\beta$-catenin has been linked to increased permeability and small-pore barrier dysfunction in vitro $[14,15]$. In mice, cytosolic truncation of the VE-cadherin gene impairs VEGF-mediated endothelial survival and angiogenesis [16]. We have previously shown that VE-cadherin is present in human placental vessels and can be perturbed, resulting in altered tracer leakage $[17,18]$.

The molecular composition of tight junctions is under immense scrutiny. So far occludin and claudins have been identified as transmembrane proteins $[19,20]$. Several signalling molecules have been described on the intracellular aspect, of which zonula occludens-1 (ZO-1) is the key signalling molecule which also mediates anchorage to actin microfilaments [21]. We have localised occludin to the paracellular clefts of conduit vessels in the normal human term placenta but found no immunostaining in exchange vessels; ZO-1 was present throughout [22].

Alterations in expression of adhesion molecules in the human placenta and in systemic vessels in diabetes are just beginning to be reported [23,24]. We have previously reported (abstract) a significant reduction in the surface expression of VE-cadherin and $\beta$-catenin of placental vessels in GDM [25]. Whether this down-regulation extends to tight junctional proteins, is associated with phosphorylation of junctional proteins and is a consequence of increased angiogenesis are still to be determined.

The present study investigates the localisation pattern of VE-cadherin, $\beta$-catenin, occludin, ZO-1 and phosphotyrosine immunoreactivity in vascular profiles in human term placentae selected at random from normal and insulin-treated GDM pregnancies. The total vessel lengths in placentae from these two groups were also estimated.

\section{Materials and methods}

Tissue selection. The study was based on pregnancies monitored at Queen's Medical Centre, Nottingham (local ethical committee approval and patient consent obtained). Patients in the experimental group $(n=5)$ were diagnosed as having GDM in the second trimester by an oral $75 \mathrm{~g}$ glucose tolerance test [26]. They were managed in accordance with established protocols. All five patients required insulin for adequate glucose control (28 weeks onwards). Patients in the control group $(n=5)$ had normal (uncomplicated) term pregnancies. Patients in both groups were of similar age, non-smokers with no history of microvascular complications, hypertension or pre-eclampsia. The birth weights of neonates, all delivered by elective Caesarean section in the last trimester, were recorded in both groups and their centiles calculated using local customised charts which made allowances for maternal size, ethnicity and parity [27]. All neonates from the GDM group were 
Table 1. Maternal, neonatal and placental characteristics of the control group: normal uncomplicated pregnancies

\begin{tabular}{lllll}
\hline Patients & $\begin{array}{l}\text { Birth } \\
\text { weight }(\mathrm{kg})\end{array}$ & Centile & $\begin{array}{l}\text { Placental } \\
\text { weight }(\mathrm{gm})\end{array}$ & $\begin{array}{l}\text { Gestational age } \\
\text { at delivery (week) }\end{array}$ \\
\hline A & 3.70 & 76 & 675 & 37 \\
B & 3.86 & 86 & 902 & 38 \\
C & 2.84 & 9 & 511 & 37 \\
D & 2.96 & 28 & 562 & 39 \\
E & 4.0 & 62 & 670 & 41 \\
\hline
\end{tabular}

macrosomic $\left(\geq 97^{\text {th }}\right.$ centile). All neonates from the normal pregnancy group had birth weights between 3-97 th centiles. The clinical details of patients are shown in (Tables 1, Table 2).

Tissue processing. Placentae were collected immediately after delivery and the umbilical cord clamped so as to prevent collapse of the vessels and minimise further blood loss from the placenta. Once in the laboratory, placental wet weights were recorded. The clamps were removed and the fetal vasculature of selected lobules of normal and GDM placentae were perfused for a 20-min equilibration period (to reverse any post-parturition hypoxic changes) with oxygenated Medium 199 according to a well-established method [1]. The maternal circulation was also independently perfused. Fetal flow was $5 \mathrm{ml} / \mathrm{min}$, maternal flow $18 \mathrm{ml} / \mathrm{min}$, fetal pressure $40-80 \mathrm{mmHg}$ and maternal pressure less than $20 \mathrm{mmHg}$. The fetal vessels were perfusion-fixed for $30 \mathrm{~min}$ with $0.4 \%$ para-formaldehyde in $0.1 \mathrm{~mol} / 1$ phosphate buffer ( $\mathrm{pH} 7.3$ ), excised and immersion-fixed for a further $120 \mathrm{~min}$. We took $2 \mathrm{~cm}^{3}$ biopsy specimens and froze them in nitrogen-cooled isopentane. Tissue biopsy specimens were also processed for transmission electron microscopy to evaluate structural integrity and any malperfusion effects. Any placentae with such deficiencies were not used for any subsequent analyses.

Antibodies. All antibodies were purchased from Sigma, St Louis, Mo., USA, unless stated otherwise. The primary antibodies used were: mouse anti-human monoclonal antibody to VE-cadherin, clone 55-7H1 (5 $\mathrm{g} / \mathrm{ml}$, Pharmigen, San Diego, Calif., USA); rabbit polyclonal antibody to $\beta$-catenin $(5 \mu \mathrm{g} /$ $\mathrm{ml})$; rabbit polyclonal antibody to ZO-1 $(10 \mu \mathrm{g} / \mathrm{ml}$, Zymed, Calif., USA); rabbit polyclonal antibody to occludin $(20 \mu \mathrm{g} / \mathrm{ml}$, Zymed) and rabbit polyclonal antibody to phosphotyrosine $(5 \mu \mathrm{g} / \mathrm{ml}$, Transduction Laboratories, Lexington, Ky., USA). Secondary antibodies were Flourescein isothiocyanate-conjugated (FITC) goat anti-mouse IgG $(11 \mu \mathrm{g} / \mathrm{ml})$ and tetramethyl rhodamine isothiocyanate (TRITC)-conjugated goat anti-rabbit $\operatorname{IgG}(50 \mu \mathrm{g} / \mathrm{ml})$.

Immunostaining. Frozen sections of placental tissue were airdried, washed in PBS ( $\mathrm{pH} 7.2$ ), permeabilized with $0.1 \%$ Triton $\times 100$ for $10 \mathrm{~min}$, blocked with $5 \%$ normal human serum (30 min, room temperature) and incubated with appropriate antibody overnight at $4{ }^{\circ} \mathrm{C}$. Sections were thoroughly washed in $\mathrm{PBS} \pm 0.1 \% \mathrm{BSA}$ and incubated with appropriate secondary antibody for $2 \mathrm{~h}$ at $37^{\circ} \mathrm{C}$.

Microscopy. Immunoreactivities were assessed using a Nikon fluorescence microscope (Nikon, Welwyn Garden City, UK) and a Leica TCS4D laser scanning confocal microscope (Leica Microsystems, Milton Keynes, UK). Optical sections (0.4-0.8 um intervals) were stored and manipulated using the accompanying software. Maximum intensity projections and single optical sections (same laser settings, same depth of sectioning) were compiled for comparing fluorescent intensities between normal and GDM immunoreactivities.

Vessel sampling. The relative incidences of vascular profiles showing different junctional immunoreactivities were estimated by applying random sampling procedures and unbiased profile counting rules. Provided that encounters between section planes and vessels occur at random orientations, these two-dimensional counts also represent the relative lengths in threedimensional immunostained regions along the entire length of the vascular bed $[28,29]$. Sections were therefore cut at different positions in haphazardly oriented tissue blocks within which the spatial arrangements of placental villous vessels are highly variable.

For sampling profiles on immunostained slides, systematic random sampling of fields of view was adopted [30]. Using the fluorescence microscope and a grid inserted into the eye-piece, the numbers of vascular profiles showing junctional immunoreactivity on section planes were counted using the unbiased 'forbidden line' counting principle [31]. These numbers were subsequently expressed as percentages of total vessels counted (all vessels, whether positive or negative for immunoreactivity). The percentage of vessels for each antibody was recorded. To maintain efficiency, we aimed at counting no more than about 200 sampling items (vascular profiles showing positive immunoreactivity) for each placenta.

Stereological estimations. To be sure that relative lengths of immunostained regions were not misinterpreted due to changes in total vessel length during insulin-treated GDM, we made

Table 2. Maternal, neonatal and placental characteristics of the study group: pregnancies complicated by insulin-treated gestational diabetes

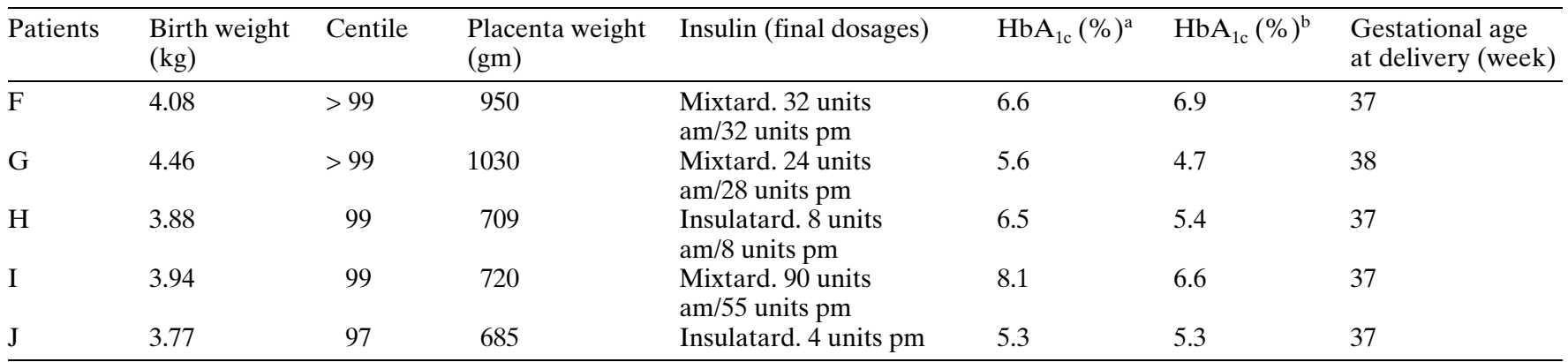

$\mathrm{HbA}_{1 \mathrm{c}}$ was measured $\mathrm{at}^{\mathrm{a}}=$ end of second trimester, ${ }^{\mathrm{b}}=$ end of third trimester. $\mathrm{HbA}_{1 \mathrm{c}}<6.8 \%$ was considered normal. Formulation of Mixtard 70:30 
stereological estimates of the total lengths of vascular beds in control and GDM placentae. To minimise shrinkage and processing distortions, toluidine blue-stained semi-thin resin sections were used. Systematic random sampling of fields was achieved using an Olympus microscope (Olympus, Denmark, A/S Silkborg, Denmark) with a motorised stage controlled by $\mathrm{x}-\mathrm{y}$ axis step motors. Selected fields were projected on to the horizontal workbench and analysed using a pair of test lattices, each of which was superimposed at random. One lattice comprised a quadratic array of test points and was used to estimate the volume density of fetal vessels within placenta [28,29]. The other comprised an unbiased counting frame [30] and was used to estimate the length density of vessels within each placenta.

The mean calibre diameter was estimated by dividing volume density by length density (to obtain vessel mean cross-sectional area) and then calculating the diameter of an equivalent circle. Total length was found by multiplying length density by placental volume, which was obtained from placental weight and an empirical estimate of tissue density $\left(1.05 \mathrm{~g} / \mathrm{cm}^{3}\right)$.

Measurements were done on placentae which had been coded, experimenters were blinded about origin of sections until after all measurements were taken and calculated.

Statistics. Group means and standard errors of means (SEMs) were calculated for each variable. For group estimates of the mean ratio between placental weight and birth weight, ratios were first calculated for each subject. Values of the percentages of vessels showing immunolocalisation for VE-cadherin, $\beta$ catenin, occludin, ZO-1 and junctional phosphotyrosine were calculated for normal and insulin-treated GDM placentae. The Mann-Whitney U test was used to compare groups and apparent differences were considered to be significant at a probability level of $p$ equal to or less than 0.05 . Correlations between selected variables and birth weight were undertaken in each group using Spearman's coefficient of rank correlation.

\section{Results}

All neonates in the insulin-treated GDM group were macrosomic $\left(\geq 97^{\text {th }}\right.$ centile) but the apparent birthweight differences between groups just failed to reach statistical significance. The mean (SEM) weight of the placenta in the GDM group was 819 (71.3) $\mathrm{g}$ whereas that in the normal group was 664 (67.3) g. This apparent difference did not achieve statistical significance. The ratio between placental weight and birth weight (the placental ratio) was similar in both groups.

Analyses of adherens junctional proteins. Vascular endothelial-cadherin and $\beta$-catenin, were immunolocalised to endothelial paracellular clefts of the placental vasculature in normal pregnancies and those complicated by insulin-treated GDM (Fig.1). Optical slicing and rotation of the sections by confocal laser scanning microscopy showed that, in both groups of placenta, VE-cadherin was localised to discrete membrane microdomains and was present through the entire extent of clefts in large vessels and in microvessels (Fig.1). A similar profile was seen for the cytoplasmic linking molecule, $\beta$-catenin (Fig. 1). The per- centage of immunoreactive vessels (Fig.2) differed, however, between the two groups. In control placentas, the proportion of vessels staining positively for VE-cadherin amounted to almost $100 \%$ and the proportion staining for $\beta$-catenin to about $94 \%$. The corresponding figures fell to $80 \%$ and $65 \%$, respectively in the GDM group ( $p<0.01$; Table 3$)$. This loss of immunoreactivity was not related to position in the vascular tree, because both large and small vessels were affected. In GDM, there was also a reduction in the intensity of immunofluorescence for both molecules (Fig. 1). Of the five placenta in the GDM group, two had a severe reduction in VE-cadherin (and $\beta$-catenin) immunoreactive vessels (Fig.2).

Analyses of tight junctional proteins. Occludin was localised only along the paracellular clefts of large vessels (arteries, veins and arterioles) of the normal human term and insulin-treated GDM placenta (Fig. 3). Microvessels in the terminal villi in both types of placentae were devoid of occludin immuno-

Fig. 1 A-F. Confocal fluorescent micrographs of human term placenta showing localisation of adherens junctional molecules, VE-cadherin and $\beta$-catenin in placentae from normal pregnancies and those complicated by insulin-treated gestational diabetes. Bar $=50 \mu \mathrm{m}$. Stem villi $=\mathrm{sv}$, intermediate vil$\mathrm{li}=\mathrm{iv}$, terminal villi $=\mathrm{tv}$. A VE-cadherin immunoreactivity in normal term placenta (patient E). Immunoreactivity is present in continuous lines corresponding to endothelial paracellular clefts of vessels. Fluorescent 'hot spots' of immunoreactivity are visible in cleft extents of longitudinal and oblique vascular profiles suggestive of VE-cadherin clustering to discrete membrane regions. B VE-cadherin in GDM placenta (patient G). Positive paracellular immunostaining can been seen in stem terminal villi. Note reduced intensity of immunostaining in these GDM vessels compared with normal placenta vessels in Fig. 1A. Both images are maximum intensity projections of 20 optical slices (total thickness $8 \mu \mathrm{m}$ ), acquired at the same laser power setting. Patient $\mathrm{G}$ is one of the two patients with pronounced reduction in number of vessels $(<60 \%)$ showing immunoreactivity to adherens junctional molecules. C Anti-VEcadherin immunoreactivity in GDM placenta (patient G). Vascular profiles showing positive paracellular immunoreactivity are interspersed with those with no immunostaining (asterisks). The latter were scored as immunonegative in this study. D Anti $\beta$-catenin immunoreactivity in normal term placenta (patient $\mathrm{E}$ ). $\beta$-Catenin can been seen in paracellular clefts of vessels in all villi. Note presence of $\beta$-catenin at the cell-cell borders of trophoblasts (arrow). E Anti $\beta$-catenin immunoreactivity in GDM placenta (patient $\mathrm{H}$ ). Micrograph shows reduced intensity of anti- $\beta$-catenin immunoreactivity compared with (Fig. 1D). Both micrographs are maximum intensity projections of 20 optical slices $(0.4 \mu \mathrm{m}$ per slice) acquired on the same laser setting. Note immunoreactivity in the overlying trophoblast layer is still present in the GDM placenta. F Anti $\beta$ catenin immunoreactivity in GDM placenta (patient I). Micrograph shows a stem villus vessel with greatly reduced anti- $\beta$ catenin immunofluorescence in paracellular clefts. Note lack of $\beta$-catenin (asterisks) in adjacent intermediate and terminal villi 


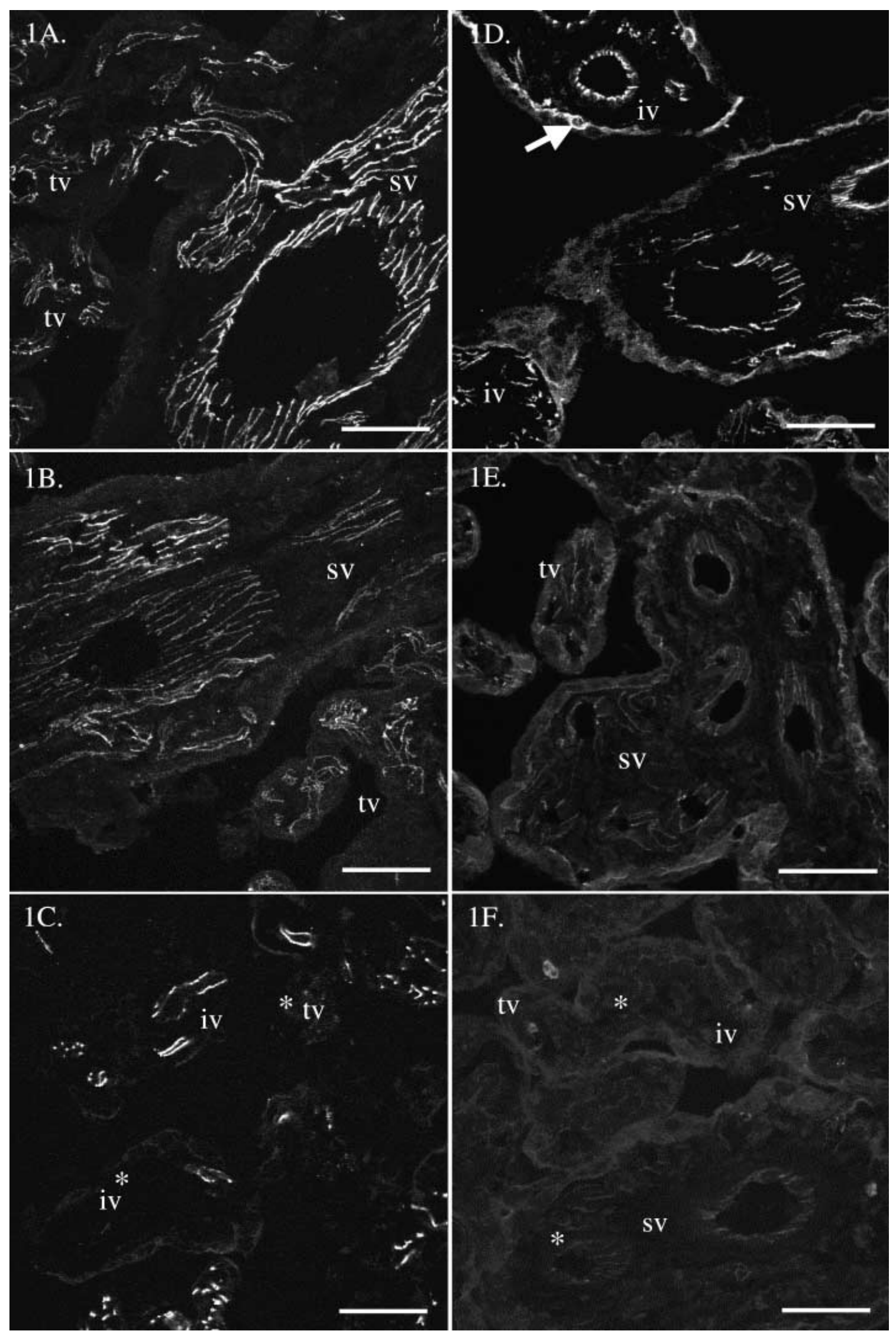


Table 3. The relative incidences of vessels immunoreactive for various junctional adhesion molecules in placentae from normal and insulin-treated GDM pregnancies

\begin{tabular}{llllll}
\hline Group & VE-cadherin & $\beta$-catenin & Z0-1 & Occludin & P-tyrosine \\
\hline Normal & $99.3(0.43)$ & $94.2(2.86)$ & $100(0.00)$ & $97.7(0.57)$ & $19.2(0.56)$ \\
GDM & $79.6(8.72)^{\mathrm{a}}$ & $64.6(3.78)^{\mathrm{a}}$ & $89.3(1.15)^{\mathrm{a}}$ & $96.0(0.27)^{\mathrm{b}}$ & $56.4(1.23)^{\mathrm{a}}$ \\
\hline
\end{tabular}

Values are group means (SEM) expressed as a percentage of vessel profiles in each study group. ${ }^{\text {a }}$ denotes significant at $p<0.01$;

${ }^{\mathrm{b}}$ denotes significant at $p<0.05$

staining (Fig. 3). A percentage of large vessels from the GDM placentae showed a reduction in the intensity of occludin immunofluorescence (Fig.3). Although the percentage of immunoreactive large vessels in the GDM and normal placenta was roughly $96 \%$ or higher, the apparent difference between groups, although small, was significant $(p<0.05$; Table 3, Fig. 2).

Zonula occludens-1, the cytoplasmic linking protein of tight junctions, was immunolocalized to the paracellular clefts of large vessels and microvessels. All the vessels analysed in the normal term placenta (Fig. 3), but only $89 \%$ of those analysed in the insulin-treated GDM placenta (Fig. 3) were immunopositive. The difference was significant $(p<0.01$; Table 3 , Fig.2). Negative vessels were not restricted to a particular size class, loss of junctional ZO-1 was seen throughout the vascular bed.

Phosphorylation of junctional proteins. The location of anti-phosphotyrosine immunoreactivity was very

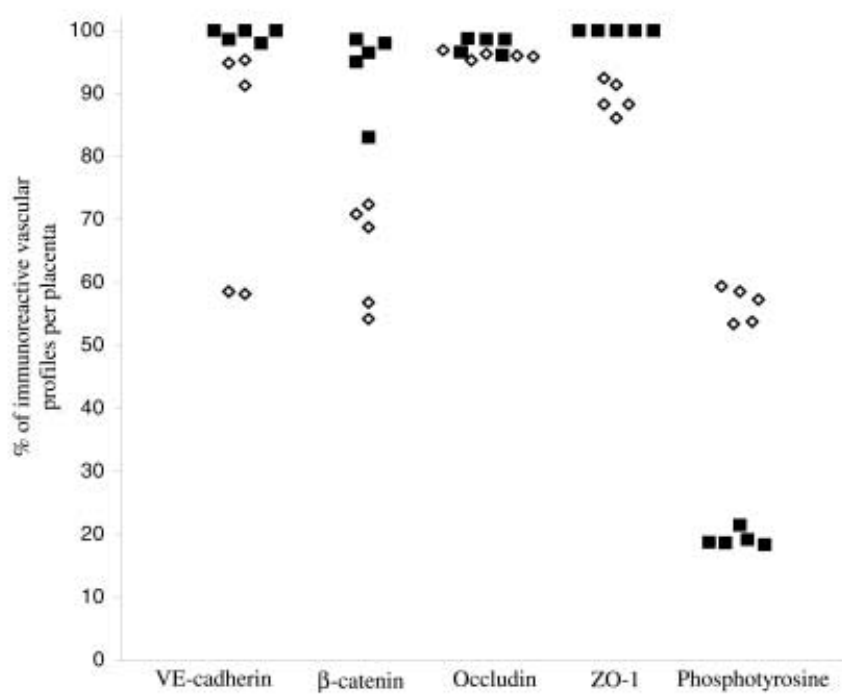

Fig. 2. Effect of gestational diabetes on junctional molecules. Scatter diagram showing percentage of human placental vascular profiles immuno-positive (at paracellular clefts) for VEcadherin, $\beta$-catenin, occludin and ZO-1 for each of the five term placentae from normal pregnancies (patients A-E) and insulin-treated GDM pregnancies (patients F-J). Percentage of vessels with anti-phosphotyrosine immunoreactivity at paracellular clefts is also shown. A systematic random sampling method was used to count the vessels. normal, $\diamond$ GDM similar to that for junctional proteins in that it was associated with paracellular clefts. In the normal human term placenta, phosphotyrosine immunoreactivity was observed as weakly fluorescent lines corresponding to the endothelial paracellular clefts in $19 \%$ of sampled vessels (Fig. 4, 2). In insulin-treated GDM placentae, phosphotyrosine immunoreactivity was seen as bright fluorescent lines corresponding to paracellular clefts in $56 \%$ of the vessels analysed, regardless of position in the vascular tree (Figs. 4, 2). There was a highly significant difference in expression of junctional anti-phosphotyrosine immunoreactivity in GDM vessels compared with normal vessels (Table 3, Fig. 2).

Fig. 3 A-F. Fluorescent micrographs of human term placenta showing immuonolocalisation of the tight junctional molecules, occludin and ZO-1, in placentae from normal pregnancies (patients $\mathbf{B}$ and $\mathbf{C}$ ) and those complicated by gestational diabetes (patient F). Bar $=50 \mu \mathrm{m}$. A Occludin in normal placenta (patient B). Confocal micrograph (single optical section, $0.4 \mu \mathrm{m}$ ) showing immunolocalisation of occludin in endothelial paracellular clefts of intermediate (iv) and stem villi (sv) vessels. Note lack of occludin (asterisk) in the capillary of an adjacent terminal villus (tv). B Single optical section showing occludin in GDM placenta (patient F). Immunolocalization of occludin is similar to that seen for normal placentae, i.e., occludin can be seen in paracellular clefts of a stem villus (sv) vessel and is absent from an adjacent capillary (*) in the terminal villi (tv). A vessel in the intermediate (iv) can now be seen as immuno-negative. There was a small but statistically significant reduction in the percentage of occludin immuno-positive vessels in GDM placentae. C Anti-occludin immunolocalisation in GDM placenta. Fluorescent micrograph showing vascular profiles in a stem villus (sv) with greatly reduced anti-occludin intensity of immunofluorescence. Note negative immunoreactivity in an adjacent intermediate villus (*). Capillaries in terminal villi (tv) are immuno-negative. D ZO-1 immunolocalisation in normal term human placenta. Confocal micrograph (single optical section, $0.4 \mu \mathrm{m}$ thick) shows anti-ZO-1 in stem (sv) and intermediate villi (iv). E Immunolocalisation of ZO-1 in GDM placenta (patient F). Confocal micrograph (single optical section, $0.4 \mu \mathrm{m}$ thick) shows ZO-1 immunolocalised at endothelial paracellular clefts of vessels in the stem (sv) and intermediate villi (iv). F Immunolocalisation of ZO-1 in GDM placenta (patient F). Confocal micrograph (single optical section, $0.4 \mu \mathrm{m}$ thick) showing reduced fluorescent intensity in vascular profiles of stem (sv) and intermediate villi (iv) compared with that in the single optical section taken from the normal placenta (Fig.3D). These GDM vessels were counted as positive vessels 


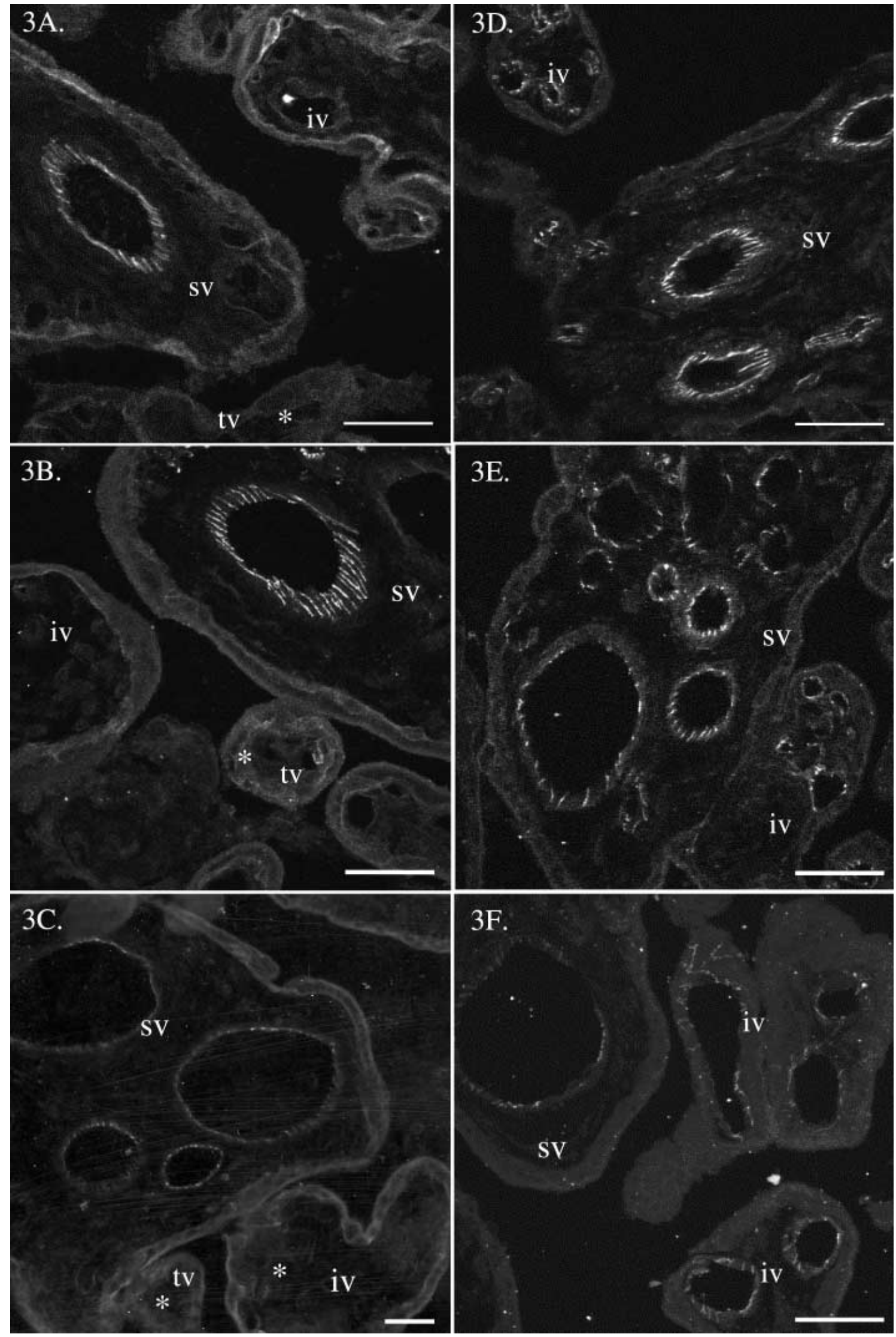



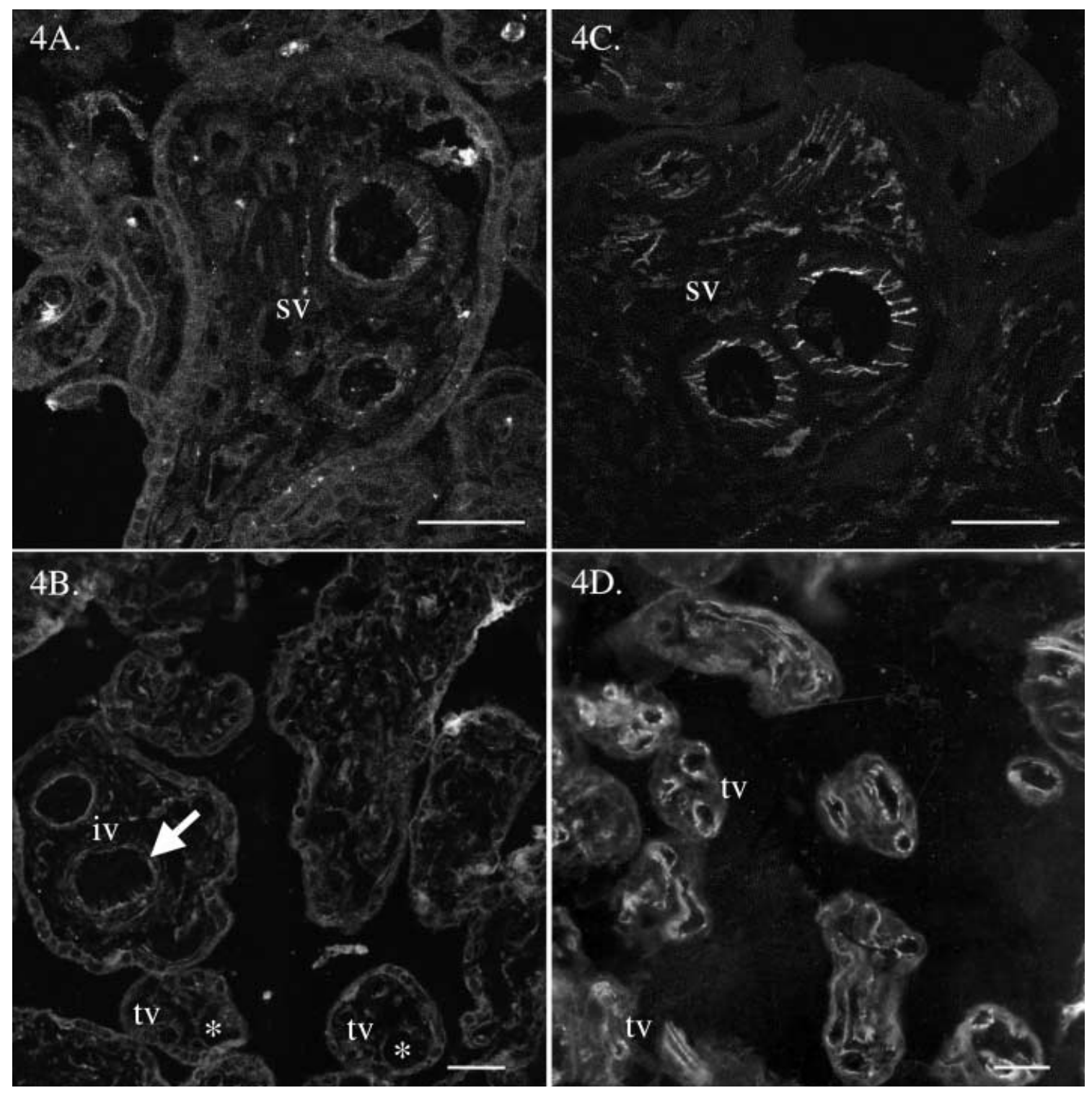

Figs. 4A-D. Anti-phosphotyrosine immunoreactivity in (A, B) normal term (patient A) and GDM placentae (C, D). A Confocal micrograph (single optical section, $0.4 \mu \mathrm{m}$ thick) shows weak immunoreactivity in vascular profiles of a stem villus (sv). Immunoreactivity to anti-phosphotyrosine was localised to endothelial paracellular clefts (similar location to junctional adhesion molecules). B Fluorescent micrograph showing reduced (arrow) immunofluorescence in an intermediate villus (iv) and negative immunoreactivity in capillaries present in terminal villi (tv, asterisks). In placentae from normal pregnancies $<19 \%$ of vessels showed paracellular immunostaining. C Micrograph from patient G. Confocal micrograph (single optical section, $0.4 \mu \mathrm{m}$ thick) showing a stem villus (sv) with paracellular anti-phosphotyrosine immunoreactivity in vascular profiles. Note the higher intensity of fluorescence in these profiles compared with that in Fig. 4A (normal placenta). D Fluorescent micrograph (from patient I) shows numerous capillaries in terminal villi (tv) with bright anti-phosphotyrosine immunoreactivity at paracellular clefts. There was a $(37 \%)$ increase in the number of vessels showing paracellular phosphotyrosine immunoreactivity in all the insulin-treated GDM placentae
Morphometric analyses. Using semi-thin resin sections, the mean (SEM) length of vessels in normal placentas was found to be $324(41.5) \mathrm{km}$ with a mean calibre diameter of 18.0 (1.6) $\mu \mathrm{m}$. These values were not significantly different in insulin-treated GDM placentae [290 (61.2) $\mathrm{km}$ and $16.2(0.9) \mu \mathrm{m}$ respectively].

Correlation of immunoreactivity with vessel lengths. A significantly higher proportion of vessels in the insulin-treated GDM group did not show immunoreactivity to antibodies against adherens or tight junctional molecules (Table 3). This loss of reactivity was seen throughout the vascular tree and not only in terminal microvessels. There was an increase in the proportion of GDM vessels showing phosphotyrosine immunoreactivity at paracellular clefts. Taken together, the differences in relative incidences of immunostained vessels represent real changes in the absolute lengths of immunostained regions along those ves- 
sels: the combined length of fetal vessels did not alter significantly in GDM placentas.

Correlation of immunoreactivity with birth weight and placental weight. Because of apparent differences in birth and placental weights, we examined whether there was any correlation between these variables and the numbers/lengths of vessels showing positive immunoreactivities. In normal placentas, Spearman's rank correlation analysis failed to find any significant correlation between birth weight and the incidences of various immunoreactivities or between placental weights and immunoreactivities. In placentae from insulin-treated GDM pregnancies, there were significant negative correlations between birth weight and phosphotyrosine immunoreactivity as well as between placental weight and phosphotyrosine immunoreactivity (correlation coefficient $=-0.90$; $p<0.05$ in both cases).

\section{Discussion}

This study shows that perturbation of adherens junctional (VE-cadherin and $\beta$-catenin) and tight junctional (ZO-1 and occludin) molecules occur in the vasculature of human placentae from pregnancies complicated by insulin-treated gestational diabetes. Statistically significant reductions were found in the percentages of vessel profiles (and, hence, proportions of total vessel lengths) immunostained for VE-cadherin, $\beta$-catenin, ZO- 1 throughout the vascular tree or immunostained for occludin in the case of large vessels. These changes were further associated with an increase in tyrosine phosphorylation at junctional regions, implicating the tyrosine kinase-signalling pathway in this perturbation. The endothelial changes we observed could be a reflection of impaired barrier function or increased angiogenesis or both of placental vessels induced by the GDM milieu. We saw, however, no difference in the absolute length of vessels in the placentae from insulin-treated GDM pregnancies.

Gestational diabetes and barrier function. Inflammatory mediators such as VEGF or histamine are reported to induce increases in vascular permeability which have been linked to the reversible redistribution of adhesion molecules from junctional regions $[14,15,32,33]$. The perturbation of junctional molecules in diabetes is, however, only just being reported. Using the diabetic rat, it has been shown [34] that VEGF could induce rapid phosphorylation of the tight junctional proteins, occludin and ZO-1. Our study showed perturbation of the adherens junctional VE-cadherin in a human disease. Vasoactive agents which are active in diabetes, such as chronic hyperglycaemia, oxygen free radicals, increased inflammatory mediators, cytokines, VEGF, hypoxia and ad- vanced glycation end-products, have been linked to a delayed and sustained increase in permeability $[5,35,36]$. The underlying cellular mechanisms, specifically the involvement of the inter-endothelial junctions, have not been explored.

The cadherin-catenin complex in endothelial cells is thought to be dynamic and capable of changing composition in relation to the microenvironment or functional status. A recent study has emphasised the role of VE-cadherin in maintaining vascular permeability in vivo: intravenous injection of an antibody mAB BV13, directed against mouse VE-cadherin, induced a concentration-dependent and time-dependent increase in vascular permeability in murine hearts and lungs, causing interstitial oedema and haemorrhage [37]. In human endothelial angiosarcoma or in hemangiomas where the vascular structures are profoundly altered, VE-cadherin is poorly expressed or lost from cell-cell contacts [13]. In our study, the reduced incidence of VE-cadherin, $\beta$-catenin and ZO-1 and the increased phosphorylation observed in paracellular clefts of GDM placental vasculature could be a consequence of inflammatory events. Perturbation of VE-cadherin by inflammatory mediators can occur in the human placenta; perfusion of human placental microvessels with $100 \mu \mathrm{mol} / \mathrm{l}$ histamine resulted in a complete loss of VE-cadherin and platelet endothelial cell adhesion molecule 1 (PECAM-1) from paracellular clefts, widening of tight junctional gaps and increased extraction of radiolabelled molecular tracers [18].

In porcine aortic endothelial cells, hyperglycaemia does elicit a dose-dependent increase in albumin permeability by activation of the protein kinase $C$ pathway [35]. Studies in our laboratory have shown that a $2 \mathrm{~h}$ hyperglycaemic insult perturbs VE-cadherin, resulting in the appearance of a discontinuous immunostaining pattern and increased permeability to 40 $000 \mathrm{M}_{\mathrm{r}}$ dextrans in HUVEC cells [38]. As stated earlier, this discontinuous staining or loss of immunofluorescence at paracellular gaps have been linked to increased permeability in numerous in vitro studies.

The association of VE-cadherin with the catenins is important. Truncation of the cytoplasmic tail of VE-cadherin results in loss of control of paracellular permeability even though the mutant molecule can still cluster at junctions and promote cell-cell attachment [39]. The association can be perturbed by inflammatory mediators; VEGF causes tyrosine phosphorylation of $\beta$-catenin, loss of $\beta$-catenin from sites of small paracellular hole formation and time-dependent and concentration-dependent increase in permeability of bovine pulmonary artery endothelial cell monolayer [15]. The loss of $\beta$-catenin from about $35 \%$ of the GDM vessels sampled in this study suggests $\beta$-catenin reorganisation in adherens junctions and barrier dysfunction in these paracellular clefts. The loss seemed to be restricted to the endothelium; 
no loss of $\beta$-catenin was seen in the overlying epithelial layer (trophoblast).

The considerable reduction in VE-cadherin and $\beta$ catenin immunoreactive vessels $(<60 \%)$ in two of the placentae from the GDM group was surprising. One of the two mothers subsequently developed post-partum Type II (non-insulin-dependent) diabetes mellitus. Gestational diabetes is well known to increase the risk of developing overt diabetes later in life [40]. In an earlier study [23] and in ongoing work, we found a severe down-regulation of VEcadherin in placentae from women with pre-gestational diabetes $(0-20 \%$ immunostained vessels, dependent on maternal microvascular complications). In the present study, the second patient who showed a pronounced decrease in immuno-staining profile had also had a previous GDM pregnancy. Both predisposition to diabetes and a number of chronic inflammatory events could therefore influence localisation and expression of endothelial junctional adhesion molecules.

Zonula occludens- 1 is involved in cadherin-based cell adhesion in non-epithelial cells by working as a cross-link between the cadherin/catenin complex and the actin-based cytoskeleton [21]. It is therefore not surprising that we found a statistically significant reduction in the number of vessels showing ZO-1 immunolocalisation along with those of VE-cadherin and $\beta$-catenin. The role of $\mathrm{ZO}-1$ in the cadherinbased cell adhesion system does not exclude its function in tight junctions. Phosphorylation of ZO-1 has been linked with decreased transcellular electrical resistance in brain endothelial cells [41].

In the human placenta, insulin-treated GDM seems to cause a large increase in tyrosine-phosphorylation of junctional proteins; both intensity of fluorescence and number of vessels (56\%) with anti-tyrosine phosphorylation immunoreactivity in clefts were increased in the GDM group. In our study, we did not attempt to isolate which of the junctional proteins were phosphorylated. Apart from the endothelial specific VE-cadherin, the presence of adhesion molecules in the overlying epithelial would have created unacceptable background levels. Furthermore, the wealth of evidence published shows that all the adhesion molecules investigated in this study are vulnerable to tyrosine-phosphorylation and their adhesive function can be modulated by this pathway. In addition, or alternately, the increased phosphorylation could also be the result of tyrosine kinase activation of insulin, IGF-1 or VEGF receptors, i.e. cell surface receptors which might also be present in paracellular membrane domains. All of these receptors are susceptible to the diabetic milieu [34, 42].

It has been recently shown [16] that transgenic mice with truncated VE-cadherin form primary tubes but not vascular networks. These findings further confirm the functional importance of VE-cadherin in vascular remodelling and angiogenesis. De-adhesion and disruption of junctional complexes have to occur before endothelial cells can detach, proliferate and migrate to form new blood vessel sprouts; junctional de-adhesion seems to be the common mechanism in loss of barrier function and angiogenesis. The potent angiogenic factor VEGF, has been shown to induce tyrosine phosphorylation of adherens junctions and allow endothelial cell motility [14]. In epithelial cells, freely available or dissociated $\beta$-catenin is reported to participate directly in the Wnt growth factor signalling cascade, translocate to the nucleus and regulate the gene expression of cellular growth and differentiation [43]. In our study, the reduction in VE-cadherin and $\beta$-catenin expression from junctional regions and the corresponding increase in tyrosine phosphorylation immunoreactivity in the GDM placental vasculature suggests a similar mechanism is operating; thus we cannot exclude the possibility that these changes could be indicative of some form of increased vascular remodelling in the GDM group. We did not observe $\beta$-catenin immunoreactivity in the nucleus of these vessels. However, immunoelectron methods may be required to confirm that this molecule is not translocated to the nucleus in these vessels.

Perturbation of the tight junctional molecule, occludin and phosphorylation of $\mathrm{ZO}-1$ in rat retina [34] have been linked to vascular leakage. The endothelial cells of the blood-retinal barrier contain fused, continuous tight junctions, the disruption of which would lead to increased permeability. In the placenta (and other systemic vessels), the tight junctions are not fused or continuous [1]. Therefore, the primary role of occludin and ZO-1 might not be in regulating permeability but in maintaining stable adhesion. Perturbation of these molecules could be indicative of labile junctions, which allow vascular remodelling. Notably, in the insulin-treated GDM placenta, junctions in large vessels (in intermediate and stem villi) also seem to be affected, implicating these vessels in vascular remodelling (and impaired barrier function). Branching angiogenesis, at the capillary level (in terminal villi), might not be the way in which the last trimester placenta grows and proliferates. It has been shown that extensive endothelial proliferation occurs at the intermediate villous level allowing the authors [44] to hypothesise that human placental angiogenesis in the last trimester occurs by increased capillary loop formation and bulging out of vessels as terminal villi.

In this study the differences in mean placental weight, length or diameter of fetal vessels in the two study groups failed to reach statistical significance, suggesting that increased angiogenesis is not a feature of insulin-treated GDM. There has been a report of increased capillary surface area in placentae from mothers with gestational diabetes [4]. There is stere- 
ological evidence that placental vessels from pre-gestational diabetes are statistically significantly increased in total volume, surface area and length [9]. The junctional adhesion molecule repertoire is, however, very different in this diabetic group [23]. From the stereological data on the surface densities of vessels and the numerical densities of capillary endothelial cells it is possible to estimate the mean surface area per endothelial squame cell. These calculations suggest that squame area does not alter substantially in pre-gestational diabetic placentae. The increases in total capillary surface area and length are therefore due to endothelial hyperplasia rather than hypertrophy. Studies in our laboratory (unpublished) have shown an increase in proliferating cell nuclear antigen (PCNA) activity in the endothelium of the insulin-treated GDM placentae (average increase of $12.4 \% ; p<0.01$ ) used in the present study. We therefore cannot exclude the possibility that increased proliferation is a feature of these GDM placentae. It is possible that in the insulin-treated GDM a steady state is maintained by equalising enhanced rates of endothelial cell repair/replacement and enhanced rates of cell damage/loss.

Women with GDM were treated with insulin for the last trimester. Although five fetuses were macrosomic despite normal matenal $\mathrm{HbA}_{1 \mathrm{c}}$ value, tight glycaemic control remains a cornerstone of management in such pregnancies, there is little evidence that accelerated fetal growth can be arrested or reversed by such a strategy $[45,46]$. Our data does not support the view that good maternal glycaemic control prevents macrosomia or the perturbed immunoreactive profile of placental vessels. Maternal $\mathrm{HbA}^{1 \mathrm{c}}$ values might not adequately reflect the quality of glycaemic control of the fetus.

Even short duration exposure to diabetes, during the vascular remodelling phase of placental growth, affects the surface expression and phosphorylation status of key adhesion molecules responsible for maintaining endothelial integrity.

Acknowledgements. The authors are grateful for support of this work by the Wellcome Trust (to L. Leach and T. M. Mayhew). T.M. Mayhew is also grateful to The Special Trustees of Nottingham University Hospitals for a grant to purchase the modified Olympus microscope for stereological analysis.

\section{References}

1. Leach L, Firth JA (1992) Fine structure of the paracellular junctions of terminal villous capillaries in the perfused human placenta. Cell Tissue Res 268: 447-452

2. Eaton BM, Leach L, Firth JA (1993) Permeability of perfused term human placental microvessels. J Physiol (Lond) 463: 141-155

3. Kuhl C, Hornes PJ, Anderson O (1985) Etiology and pathophysiology of gestational diabetes mellitus. Diabetes 34 (Suppl. 2): 66-70
4. Teasdale F (1981) Histomorphometry of the placenta of the diabetic women: class A diabetes mellitus. Placenta 2: 241-252

5. Tooke JE (1995) Microvascular function in human diabetes. A physiological perspective. Diabetes 44: 721-726

6. Sander B, Larsen M, Engler C, Lund-Andersen H, Parving HH (1994) Early changes in diabetic retinopathy: capillary loss and blood-retina barrier permeability in relation to metabolic control. Acta Opthalmol Scand 72: 553-559

7. Jones CJP, Fox H (1976) Placental changes in gestational diabetes. An ultrastructural study. Obstet Gynaecol 48: 274-280

8. Teasdale F (1983) Histomorphometry of the human placenta in class B diabetes mellitus. Placenta 4: 1-12

9. Mayhew TM, Sørensen FB, Klebe JG, Jackson MR (1994) Growth and maturation of villi in placentae from well-controlled diabetic women. Placenta 15: 57-65

10. Pappenheimer JR, Renkin EM, Borrero LM (1951) Filtration, diffusion and molecular seiving through peripheral capillary membranes. A contribution to the pore theory of capillary permeability. Am J Physiol 167: 13-46

11. Firth JA, Bauman KF, Sibley CP (1983) The intercellular junctions of guinea-pig placental capillaries: a possible structural basis for endothelial solute permeability. J Ultrast Res 85: 45-57

12. Shapiro L, Fannon AM, Kwong PD et al. (1995) Structural basis of cell-cell adhesion by cadherins. Nature 374: 327-337

13. Dejana E (1996) Endothelial adherens junctions: Implications in the control of vascular permeability and angiogenesis. J Clin Invest 98: 1949-1953

14. Esser S, Lampugnani MG, Corada M, Dejana E, Risau W (1998) Vascular endothelial growth factor induces VEcadherin tyrosine phosphorylation in endothelial cells. J Cell Sci 111: 1853-1865

15. Cohen AW, Carbajal JM, Schaeffer Jr RC (1999) VEGF stimulates tyrosine phosphorylation of $\beta$-catenin and small pore endothelial barrier dysfunction. Am J Physiol 277: H2038-H2049

16. Carmeliet P, Lampugnani MG, Moons L et al. (1999) Targeted deficiency or cytosolic truncation of the VE-cadherin gene in mice impairs VEGF-mediated endothelial survival and angiogenesis. Cell 98: 147-157

17. Leach L, Clark P, Lampugnani MG, Arroyo AG, Dejana E, Firth JA (1993) Immunoelectron characterisation of the inter-endothelial junctions of human term placenta. J Cell Sci 104: 1073-1081

18. Leach L, Eaton BM, Westcott EDA, Firth JA (1995) Effect of histamine on endothelial permeability and structure and adhesion molecules of the paracellular junctions of perfused human term placental microvessels. Microvasc Res 50: 323-337

19. Furuse M, Hirase T, Itoh M et al. (1993) Occludin: a novel integral membrane protein localizing at tight junctions. J Cell Biol 123: 1777-1788

20. Furuse M, Fujita K, Hiiragi T, Fujimoto K, Tsukita S (1998) Claudin-1 and -2: novel integral membrane proteins localizing at tight junctions with no sequence similarity to occludin. J Cell Biol 141: 1539-1550

21. Itoh M, Nagafuchi A, Moroi S, Tsukita S (1997) Involvement of ZO-1 in cadherin-based cell adhesion through its direct binding to alpha catenin and actin filaments. J Cell Biol 138: 181-192

22. Leach L, Lammiman MJ, Babawale MO et al. (2000) Molecular organisation of tight and adherens junctions in the human placental vascular tree. Placenta 21: 547-557 
23. Babawale MO, Lammiman MJ, James DK, Mayhew TM, Leach L (1998) Junctional adhesion molecules in long duration and gestational diabetes. A confocal study of the placental microvessels. In: Carpentier PH, Vicaut E, Guilmot JL (eds), 20 th European Conference on Microcirculation. Monduzzi Editore, Bologna pp 59-62

24. Antonetti DA, Barber AJ, Khin SK, Lieth E, Tarbell JM, Gardner TW (1998) Vascular permeability in experimental diabetes is associated with reduced endothelial occludin content. Diabetes 47: 1953-1959

25. Lovat S, Simmonds MJR, Babawale MO, Mayhew TM, James DK, Leach L (1999) The effect of gestational diabetes on adhesion molecules regulating the placental endothelial barrier. J Soc Gynaecol Investig 6: 418 (Abstract)

26. Puavilai G, Chanprasertyotin S, Sriphrapradaeng A (1999) Diagnostic criteria for diabetes mellitus and other categories of glucose intolerance: 1997 criteria by expert committee on the diagnosis and classification of diabetes mellitus (ADA), 1998 WHO consultation criteria and 1985 WHO criteria. World Health Organisation. Diabetes Res Clin Pract 44: 21-26

27. Gardosi J (1997) Customized growth curves. Clin Obstet Gynecol 40: 715-722

28. Mayhew TM (1992) A review of recent advances in stereology for quantifying neural structure. J Neurocytol 21: 313-328

29. Howard CV, Reed M (1998) Unbiased Stereology. ThreeDimensional Measurement in Microscopy. Bios Scientific, Oxford

30. Gundersen HJG, Jensen E (1987) The efficiency of systematic sampling in stereology and its prediction. J Microsc 147: 229-263

31. Gundersen, HJG (1986) Stereology of arbitrary particles: a review of unbiased number and size estimators. J Microsc 143: 3-45

32. Kevil CG, Payne DG, Mire E, Alexander, JS (1998) Vascular permeability factor/vascular endothelial cell growth factor-mediated permeability occurs through disorganisation of endothelial junctional proteins. J Biol Chem 273 (24): 15099-15103

33. Andropilou P, Navarro P, Zanetti A, Lampugnani MG, Dejana E (1999) Histamine induces tyrosine phosphorylation of endothelial cell-cell adherens junctions. Arterioscler Thromb Vasc Biol 19: 2286-2297

34. Antonetti DA, Barber AJ, Hollinger LA, Wolpert EB, Gardner TW (1999) Vascular endothelial growth factor induces rapid phosphorylation of tight junction proteins oc- cludin and zonula occluden 1: A potential mechanism for vascular permeability in diabetic retinopathy and tumors. J Biol Chem 274: 23463-23467

35. Hempel A, Maasch C, Heintze U et al. (1997) High glucose concentrations increase endothelial cell permeability via activation of protein kinase $\mathrm{C}$ alpha. Circ Res 81: 363-371

36. Wautier JL, Zoukourian C, Chappey O et al. (1996) Receptor-mediated endothelial cell dysfunction in diabetic vasculopathy: soluble receptor for advanced glycation end products blocks hyperpermeability in diabetic rats. J Clin Invest 97: 238-243

37. Corada M, Mariotti M, Thurston G et al. (1999) VE-cadherin is an important determinant of microvascular permeability in vivo. Proc Natl Acad Sci USA 96: 9815-9820

38. Payne, B. A. I. (1999) Short duration exposure to high glucose alters distribution of VE-cadherin and F-actin in human endothelial cell. J Anat (in press- Abstract)

39. Navarro P, Caveda L, Breviario F et al. (1998) Catenin-dependent and -independent functions of vascular endothelial cadherin. J Biol Chem 270: 30965-30972

40. O'Sullivan JB (1978) Gestational diabetes: factors influencing the rates of subsequent diabetes. In: Sutherland HW, Stowers JM (eds) Carbohydrate Metabolism in Pregnancy and the Newborn. Springer-Verlag, New York, pp 425-435

41. Staddon JM, Herrenknecht K, Smales C, Rubin LL (1995) Evidence that tyrosine phosphorylation may increase tight junction permeability. J Cell Sci 108: 609-619

42. Desoye G, Hartmann M, Jones CJ et al.(1997) Location of insulin receptors in the placenta and its progenitor tissues. Micros Res Tech 38: 63-75

43. Ben-Ze'ev A, Geiger B (1998) Differential molecular interactions of beta-catenin and plakoglobin in adhesion, signalling and cancer. Curr Opin Cell Biol10: 629-639

44. Kaufman P, Bruns U, Leiser R, Luckhardt M, Winterhager E (1985) The fetal vascularization of term human placental villi. II. Intermediate and terminal villi. Anat Embryol 173: 203-214

45. Konkov E, Young P, Lowy C (1997) The effect of maternal glycaemic control on fetal growth in diabetic pregnancies. Am J Pathol 14: 547-552

46. Garner P, Okuri N, Keely E et al. (1997) A randomised controlled trial of strict glycaemic control and tertiary level obstetric care versus routine obstetric care in the management of gestational diabetes: a pilot study. Am J Obstet Gynecol 177: 190-195 